\title{
Quelle géographie des transitions agricoles en France? Une approche exploratoire à partir de l'agriculture biologique et des circuits courts dans le recensement agricole 2010
}

\author{
Michaël Bermond ${ }^{1, *}$, Pierre Guillemin ${ }^{1}$ et Gilles Maréchal ${ }^{2}$ \\ ${ }^{1}$ UMR CNRS 6590 Espaces et sociétés, Université de Caen Normandie, Maison de la Recherche en Sciences Humaines, \\ Esplanade de la Paix, 14032 Caen cedex, France \\ ${ }^{2}$ Bureau d'étude Terralim, Chercheur associé UMR CNRS 6590 Espaces et Sociétés, 4 rue Surcouf, 35740 Pacé, France
}

\begin{abstract}
Résumé - À partir du recensement agricole 2010, l'article explore une méthodologie pour délimiter un champ statistique des exploitations agricoles en transition agro-écologique dans le contexte métropolitain français. Ce travail débouche sur une proposition de typologie d'exploitations combinant le mode de production (biologique ou non), le mode de commercialisation des produits et la part du chiffre d'affaire réalisée en circuit court. Après une caractérisation socio-économique des différents types d'exploitation, l'analyse propose une cartographie à échelle fine des combinaisons géographiques d'agriculture en transition pour le territoire métropolitain français, ouvrant une discussion sur les facteurs socio-territoriaux favorables à l'émergence de tel type de transition plutôt que tel autre. Au final, la géographie que dessine cette typologie d'ensemble des agricultures en transition emprunte certes des éléments explicatifs au contexte géo-agronomique des exploitations. Mais elle semble également indiquer que la nature du contexte socio-territorial dans lequel s'insèrent les exploitations infléchit les formes de transition agricole. Les espaces ruraux les moins dynamiques et les plus marqués par l'héritage du modèle productiviste restent dans leur grande majorité les plus résistants à l'émergence des formes de transition considérées dans cet article. La proximité géographique avec des espaces sociaux plus aisés constituerait un facteur favorable au développement des exploitations en transition agro-écologique.
\end{abstract}

Mots clés : agriculture de transition / exploitation agricole / recensement agricole / espaces ruraux / France

\begin{abstract}
Which geography of agricultural transitions in France? An exploratory approach from organic farming and short food supply chains in the $\mathbf{2 0 1 0}$ agricultural census. Based on the agricultural census of 2010, this article explores a methodology to identify a statistical field of farms in agro-ecological transition in the French metropolitan context. The work leads to a proposition of a typology of farming systems where the mode of production (organic or not), product marketing, and turnover percentage from direct sales channels are combined. After a socio-economic characterization of the different types of farms, the analysis proposes a fine-scale mapping of geographic combinations of agriculture in transition in Metropolitan France, thereby starting a discussion on the socio-territorial factors in favor of the emergence of one type of transition over another. In the end, the geography deduced by this overall typology of agriculture in transition certainly borrows from explanatory elements of the geo-agronomic context of farms. But it also seems to indicate that the nature of the socio-territorial context in which farms operate influences the forms of agricultural transition. Rural areas that are least dynamic and most marked by the legacy of the productivist model remain in their majority the most resistant to the emergence of forms of transition discussed in this article. Geographical proximity to more affluent social spaces would be a favorable factor for the development of farms in agro-ecological transition.
\end{abstract}

Keywords: transitional farming / farming system / agricultural census / rural areas / France

\footnotetext{
* Auteur de correspondance $:$ michael.bermond@unicaen.fr
} 


\section{Introduction : enjeux et problématique}

Alors qu'elle comptait 2,3 millions d'exploitations agricoles en 1955, la France n'en compte plus qu'environ 516000 en 2010. Forte d'une population active agricole, familiale et salariée de 6,2 millions de personnes en 1955, soit $31 \%$ de l'emploi total en France (Desriers, 2007), cette part tombe en 2010 à $3 \%$ avec 966000 personnes travaillant régulièrement sur les exploitations. Effacement démographique et réduction des emplois agricoles sont une tendance lourde bien connue, conséquences des gains croissants de productivité du travail et de l'intégration accélérée de l'économie agricole dans le mode de production capitaliste (Tavernier et al., 1972 ; Kroll, 1990).

Jusqu'à la fin des années 1970, les transferts de maind'œuvre de l'agriculture vers les autres secteurs économiques créent une baisse des emplois agricoles qui semble faire consensus. La poursuite de cette baisse, dans un contexte désormais marqué par la crise de l'emploi et la précarisation du travail, interroge sur la soutenabilité d'un modèle industriel de développement agricole valorisant désormais davantage le capital que le travail. De fait, l'entrée en crise du «modèle» productiviste agricole au regard de ses dérives économiques, sociales et environnementales débouche, dès la fin des années 1980, sur l'émergence de formes d'agriculture plus durables dont l'une des caractéristiques serait non seulement de mieux préserver les ressources environnementales mais aussi de mieux valoriser le travail et l'emploi (Garambois, 2011; Massis et Hild, 2016).

Accompagner les agriculteurs vers des formes de transition agro-écologique est désormais un enjeu politique largement institutionnalisé, inaugurant des changements significatifs dans les pratiques et les modes de production, depuis les formes les moins poussées (réduction des intrants de synthèse sans remise en cause des itinéraires techniques «de référence») aux formes les plus poussées reposant sur les principes de l'agrobiologie et des services écosystémiques. Certains agriculteurs optent pour des systèmes agro-écologiques combinant à la fois production biologique et commercialisation en circuit court, même si la transition agroécologique ne s'y réduit pas. En effet, de nombreux travaux de recherche issus de différentes disciplines rendent compte de la diversité des formes d'agriculture durable (Féret et Douguet, 2001 ; Laurent et al., 2003; Therond et al., 2017 ; Plumecocq et al., 2018) et étudient la durabilité de modèles alternatifs (réseau agriculture durable, agriculture biologique, agriculture multifonctionnelle, agriculture de conservation...). Pendant qu'une partie de la recherche agronomique s'intéresse aux pratiques nouvelles dites à plus haute performance environnementale de systèmes productifs utilisant de manière plus efficiente les fonctions écologiques des agrosystèmes (Griffon, 2013 ; Elzen et al., 2017), certains agro-économistes revisitent les rapports historiquement construits entre organisation productive à l'échelle des exploitations et organisation logistique des filières afin d'identifier les verrous et leviers d'une transition vers des agricultures plus durables (Fares et al., 2012). Les sociologues, quant à eux, analysent les conditions d'émergence de mobilisation d'acteurs (agricoles ou non) favorables aux processus d'écologisation de l'agriculture (Lamine et Bellon, 2009; Deverre et Lamine, 2010; Chantre et Cardona, 2014).
Plus rares sont néanmoins les travaux de géographes éclairant la diffusion spatiale des exploitations agricoles en transition. La littérature scientifique - avant tout anglosaxonne - qui s'est développée depuis quelques années autour d'une «nouvelle géographie alimentaire» (Wiskerke, 2009; Lamine et al., 2012) s'est d'abord intéressée aux démarches initiées dans les villes (Mansfield et Mendes, 2013), en interrogeant préférentiellement les conditions d'émergence de politiques alimentaires urbaines dans certaines métropoles (Renting et Wiskerke, 2010; Lamine et al., 2012), avant de questionner plus largement le rôle des interfaces rural-urbain dans ces dynamiques (Sonnino, 2014 ; Moragues-Faus, 2016). Peu de travaux proposent une lecture géographique et cartographique des formes d'agriculture en transition à l'échelon d'un pays.

La présente contribution entend dresser les contours d'une géographie des exploitations agricoles engagées sur la voie d'une transition écologique pour le territoire métropolitain français. Précisons d'emblée que le champ d'étude des exploitations agricoles en transition sera ici limité aux exploitations pratiquant l'agriculture biologique et/ou les circuits courts de commercialisation, deux dimensions considérées comme structurantes mais non exclusive de l'agroécologie (Maréchal et Spanu, 2010). Partant de cette hypothèse, et s'inspirant du cadre conceptuel des systèmes agroécologiques territorialisés (SAET) où s'articulent ressources matérielles de l'exploitation, filière et territoire (Duru et al., 2014), la démarche méthodologique consiste à proposer, à partir du Recensement agricole 2010, une typologie spatialisée des exploitations en transition selon les critères définis. La cartographie établie à l'échelon cantonal interroge alors le rôle des contextes socio-territoriaux (Wezel et al., 2016) dans l'agencement spatial, variable, des formes de transition. Dans la première partie de cet article, une méthode exploratoire délimite un champ statistique des exploitations agricoles en transition au sein duquel il est possible de différencier des types plus ou moins marqués par l'écologisation des pratiques agricoles. La deuxième partie caractérise le profil socio-structurel des différents types d'exploitations identifiés, et souligne l'intensité plus forte en main-d'œuvre des exploitations en transition, comparées aux exploitations conventionnelles. La troisième et dernière partie montre que les différents types d'exploitation en transition se combinent géographiquement de manière variable selon les cantons, ouvrant une discussion sur les facteurs socio-territoriaux favorables à l'émergence de tel type plutôt que tel autre.

\section{Les exploitations agricoles en transition: une approche statistique exploratoire à partir du Recensement agricole 2010}

Peut-on délimiter un champ statistique et géographique des exploitations agricoles «en transition» pour la France métropolitaine? À partir de quelle source de données et sur la base de quels critères? Souvent décrié en tant qu'appareil statistique au service d'une vision productiviste du développement agricole (Rémy, 1990), le recensement agricole reste pourtant un outil de connaissance sur l'organisation des exploitations agricoles en France. Paradoxalement, ce n'est pas l'outil le moins intéressant pour esquisser les contours 
d'une agriculture en transition, à condition de rompre pour une part avec les usages et les catégories d'analyse dominantes habituellement utilisées pour décrire les exploitations agricoles. Il permet notamment d'explorer deux hypothèses fortes.

La première porte sur le rôle moteur de la production agricole. Elle postule que ce secteur est déterminant pour accompagner une transition s'exprimant dans les secteurs de la transformation, de la distribution et des services et fournitures associés. L'observation des dynamiques territoriales dans l'Ouest de la France, et plus généralement au nord de la Loire, révèle que, dans ces régions où un type de production (élevage, céréales) dessine l'essentiel du paysage agricole, c'est la faiblesse d'une offre diversifiée qui constitue le facteur limitant. Cette assertion est sans doute discutable dans les régions au sud de la Loire où la diversité de l'offre est plus prononcée, la pratique des circuits courts intégrée de plus longue date, avec des effets concurrentiels plus aigus.

La seconde hypothèse part de l'idée que, parmi les données disponibles au sein du Recensement agricole 2010 (RA 2010), trois variables sont révélatrices du niveau de transition des exploitations: le mode de production biologique, le mode de commercialisation (circuit court/circuit long) et la part des circuits courts dans le chiffre d'affaire de l'exploitation. Ces trois variables ne peuvent, à elles seules, circonscrire le champ des exploitations en transition, mais permettent une première approche ni exhaustive, ni définitive. Des travaux récents rappellent bien que la caractérisation socio-économique des formes d'agriculture durable repose sur des approches bio-techniques et socio-techniques plus larges que celles retenues ici (Plumecocq et al., 2018), notamment pour la caractérisation de l'agriculture de conservation (Dugué et al., 2015), ou encore des systèmes herbagers autonomes et économes (Garambois, 2011). Il existe d'autres formes d'agriculture en transition qui ne sont pas prises en compte ici, comme par exemple l'agroforesterie ou la permaculture. Toutefois, parmi l'ensemble des données disponibles à petite échelle, nous faisons l'hypothèse que les trois indicateurs retenus constituent des lignes de force structurant significativement un champ des exploitations en transition.

Grâce à l'approche méthodologique développée, on peut segmenter le champ des 516152 exploitations agricoles recensées en France métropolitaine en 2010 selon la combinaison des trois variables précitées. Cette typologie résulte d'une partition emboîtée et hiérarchisée, comme le suggère l'arbre de classification associé à la figure 1: les exploitations sont d'abord divisées en deux groupes selon qu'elles sont converties ou non à l'agriculture biologique. Chacun des deux groupes est ensuite divisé en deux sousensembles selon les modes de commercialisation (circuit long versus circuit court). Enfin, une dernière distinction est opérée au sein des exploitations pratiquant les circuits courts selon l'importance que ces circuits tiennent dans la formation du chiffre d'affaires (moins de $10 \% ; 10$ à $50 \% ; 50$ à $75 \% ; 75 \%$ et plus). Au final, six groupes d'exploitations sont identifiés :

- type A : les exploitations conventionnelles exclusivement intégrées dans des circuits longs de commercialisation, représentant $80,1 \%$ du total des exploitations métropolitaines en 2010 ;
- type B : les exploitations conventionnelles réalisant moins de $50 \%$ de leur chiffre d'affaires en circuit court, regroupant $7,4 \%$ du total des exploitations;

- type C: Les exploitations conventionnelles avec un recours important au circuit court (au moins $50 \%$ du chiffre d'affaires) rassemblant $8,9 \%$ du total des exploitations ;

- type D: les exploitations biologiques exclusivement intégrées dans des circuits longs de commercialisation ( $1,7 \%$ des exploitations) ;

- type E: les exploitations biologiques réalisant moins de $50 \%$ de leur chiffre d'affaires en circuit court $(0,7 \%$ des exploitations);

- type F : les exploitations biologiques recourant au circuit court de commercialisation pour au moins $50 \%$ de leur chiffre d'affaires, soit $1,2 \%$ du total en 2010.

Les exploitations des types C, D, E, F seront ici considérées comme celles délimitant le champ des exploitations en transition. Le seuil de $50 \%$ de chiffre d'affaires réalisé en circuits courts a été choisi car, lorsque le poids de cette commercialisation augmente, certaines pratiques culturales peuvent évoluer, par exemple vers la réduction d'intrants (Aubry et al., 2011). Selon cette approche, les exploitations en transition représenteraient environ $12,5 \% \mathrm{du}$ total des exploitations en France métropolitaine en 2010, soit un peu plus de 54000 exploitations. Le parti a été pris de maintenir hors du champ de l'agriculture en transition les exploitations du type $B$ en raison du poids hétérogène des circuits courts.

\section{Caractérisation socio-économique des exploitations en transition}

La typologie proposée peut être lue comme un gradient d'écologisation des pratiques agricoles, du plus faible pour le type A (conventionnel en circuit long) au plus fort pour le type $\mathrm{E}$ (exploitations biologiques fortement tournées vers les circuits courts de commercialisation). Ce gradient d'écologisation des pratiques structure la pyramide des âges des chefs d'exploitation, ainsi que leur niveau de formation générale (Fig. 1). On observe en effet que la part des chefs d'exploitation de moins de 40 ans augmente globalement avec le degré d'écologisation des pratiques agricoles $(30 \%$ pour le type $\mathrm{F}$ contre $16 \%$ pour le type A). On observe aussi une progression du niveau de formation générale chez les agriculteurs biologiques, conformément aux résultats d'autres travaux (Mahé et Lerbourg, 2012). La part des diplômés du supérieur atteint $29 \%$ chez les exploitants biologiques fortement insérés dans les circuits courts de commercialisation, soit trois fois plus que la moyenne des chefs d'exploitation. De même, une plus forte commercialisation en circuit court est corrélée avec la féminisation des responsables d'exploitation (respectivement 25 et $27 \%$ de femmes parmi les chefs d'exploitation des types $\mathrm{C}$ et $\mathrm{F}$ contre $23 \%$ en moyenne) (Dufour et Giraud, 2012).

La répartition des exploitations selon leur orientation technico-économique (OTEX) et leur dimension économique dessinent des univers socioprofessionnels sensiblement différents selon les types (Fig. 2). À l'image de leurs homologues en système conventionnel (type A), les exploitants biologiques en circuit long (type $\mathrm{D}$ ) restent surtout positionnés sur des 


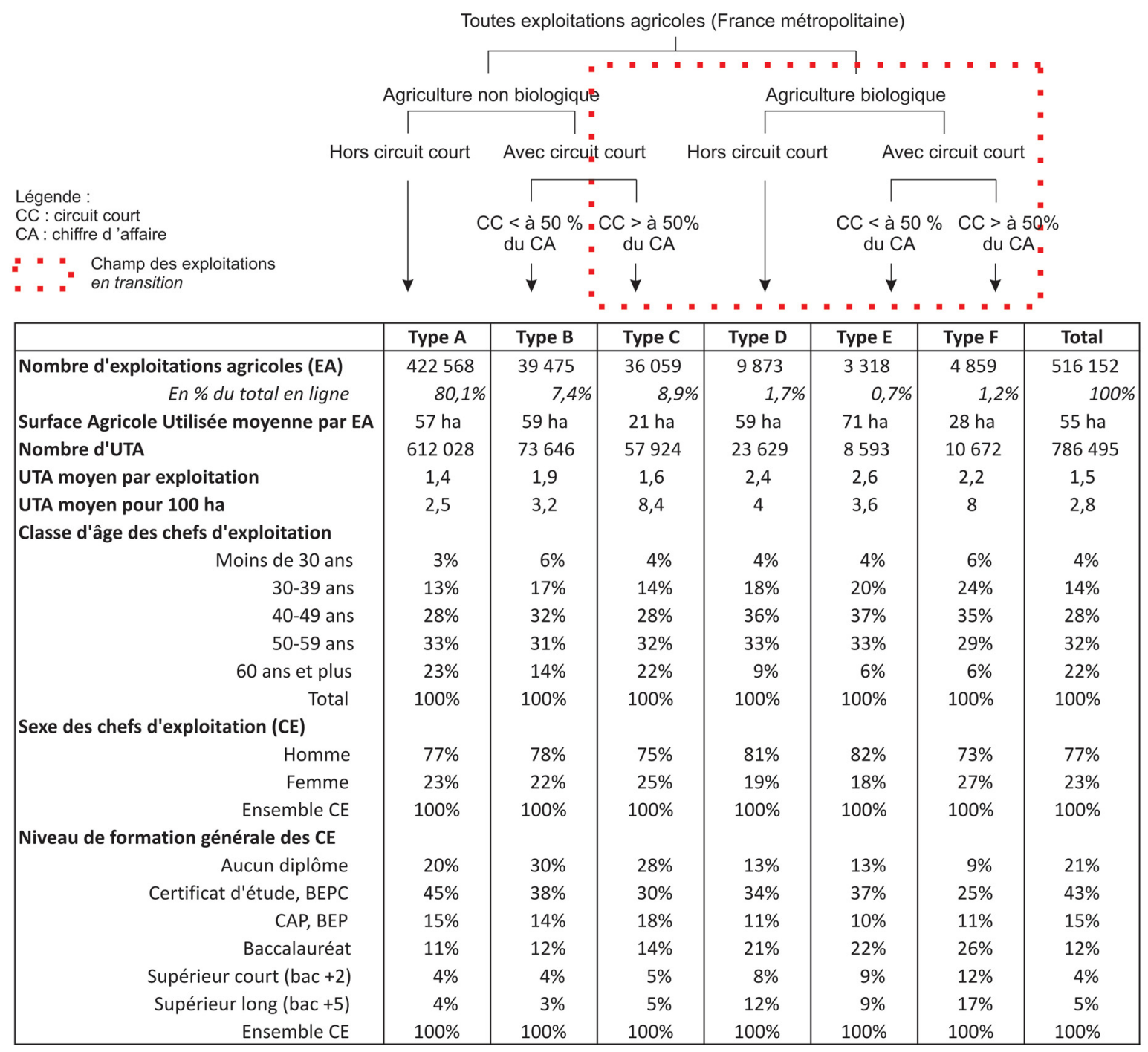

Source : AGRESTE - Recensement agricole 2010 - CASD.

Fig. 1. Caractéristiques sociodémographiques des exploitations agricoles en transition en France métropolitaine en 2010.

Fig. 1. Sociodemographic characteristics of farms in transition in metropolitan France in 2010.

produits finis adaptés à une production et une distribution de masse (grandes cultures, élevage bovin), à la différence près qu'une exploitation sur trois est ici spécialisée en viticulture. Ils travaillent sur des exploitations de taille foncière et de dimension économique en moyenne plus grandes que celles des exploitations conventionnelles. Les exploitations biologiques pratiquant un peu de circuit court (type E) suivent un profil en OTEX et en dimension économique proches également des systèmes de production conventionnels où la recherche de productivité adaptée à des systèmes alimentaires globalisés se combine à des formes de diversification basées sur l'économie locale. Une plus forte implication dans les circuits courts de commercialisation débouche a contrario sur la mise en place d'exploitations de plus petite dimension (types $\mathrm{C}$ et $\mathrm{F}$ ), avec pour objectif une meilleure efficacité économique par unité de production. Au-delà de $50 \%$ du chiffre d'affaires réalisé en circuit court, la part des exploitations maraîchères-horticoles augmente significative- ment, comme celles en fruits et autres cultures permanentes, ou en petits élevages (ovins, caprins).

La figure 1 souligne une intensité du travail plus élevée sur les exploitations en transition comparativement aux exploitations conventionnelles. Le nombre d'Unités de Travail Annuel (UTA) par exploitation - ratio classiquement utilisé pour mesurer l'intensité du travail dont la valeur 1 équivaut au travail d'une personne occupée à temps complet à des travaux agricoles pendant une année- s'élève en moyenne à 1,9 pour les exploitations en transition, contre 1,5 pour les exploitations conventionnelles. Le ratio UTA/ exploitation atteint sa valeur la plus élevée pour les exploitations biologiques pratiquant partiellement les circuits courts (type E). Les exploitations réalisant plus de $50 \%$ de leur chiffre d'affaires en circuits courts restent celles qui valorisent le mieux la main-d'œuvre par unité de surface agricole (au moins 8 UTA pour 100 ha pour les types $\mathrm{C}$ et $\mathrm{F}$, contre environ 4 pour les autres formes de transition). Ces 


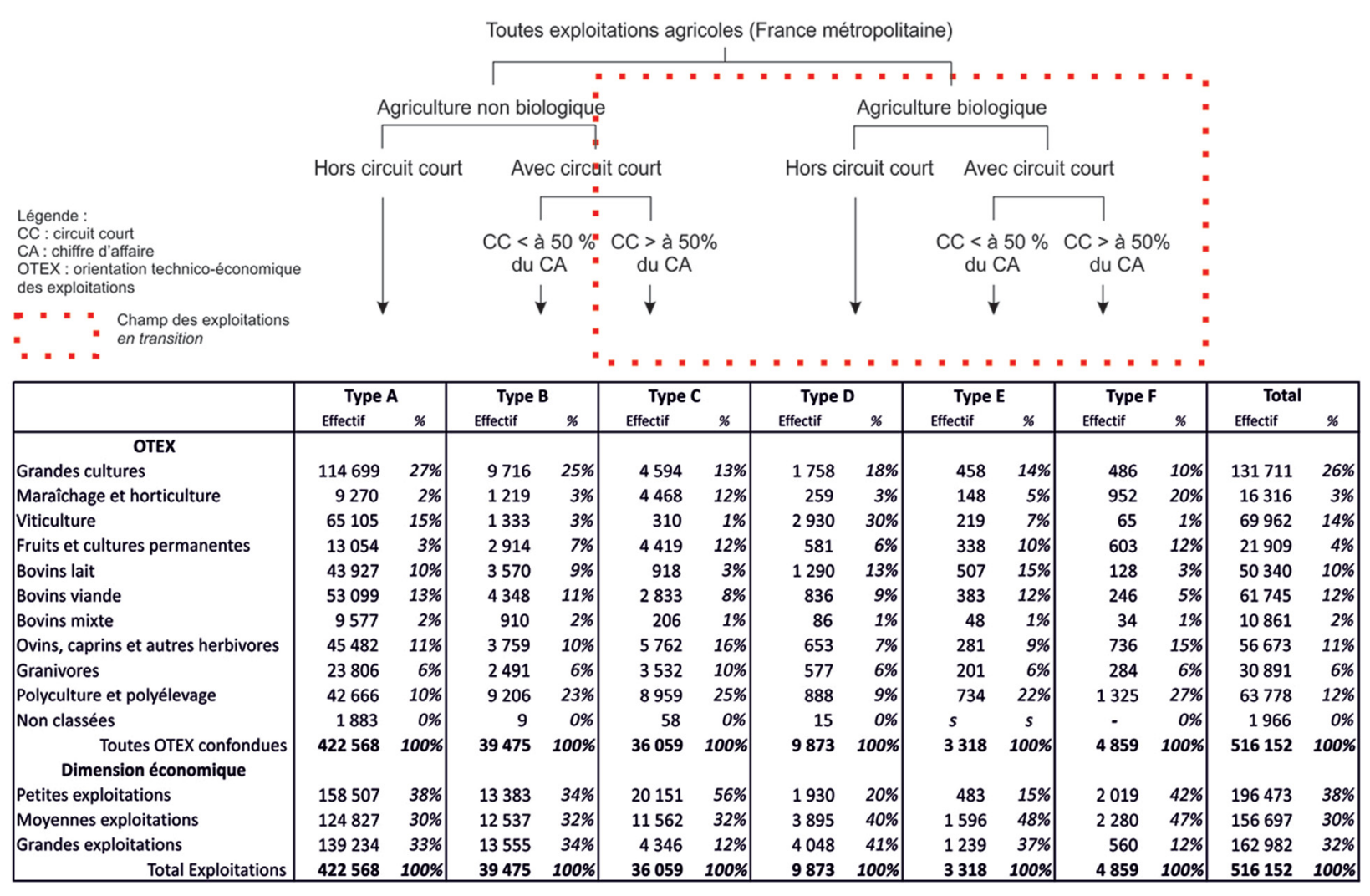

Source : AGRESTE - Recensement agricole 2010 - CASD

Fig. 2. Orientations technico-économiques et dimensions économiques des types d'exploitations agricoles en transition en 2010.

Fig. 2. Techno-economic orientations and economic dimensions of the types of farms in transition in 2010.

résultats vont dans le sens d'une meilleure contribution des formes en transition au maintien des actifs en agriculture (Bâ et al., 2016; Dedieu et al., 2017; Levaux et Genty, 2015; Massis et Hild, 2016; Roy et Teyssier d'Orfeuil, 2016). À ce stade, il serait intéressant d'analyser la structure du collectif de travail selon les statuts d'activité de la main-d'œuvre (Legagneux et Olivier-Salvagnac, 2017) pour mieux apprécier la soutenabilité sociale des formes de transition.

$\mathrm{Au}$ regard de leurs caractéristiques sociodémographiques assez différenciées, les types $\mathrm{C}$ et $\mathrm{D}$ ne concernent pas tout à fait les mêmes profils sociaux. Nous faisons l'hypothèse que ces deux groupes seraient marqués par des trajectoires d'entrée dans l'agriculture alternative bien différenciées. Les caractéristiques sociodémographiques des agriculteurs/trices du type $\mathrm{F}$ renverraient aux modes d'entrée dans l'agriculture alternative de jeunes diplômés militants en reconversion professionnelle décrits par Samak (2016). Le type C incarne plutôt des formes de « résistances paysannes» ou d'appropriation plus populaires des alternatives agricoles, contrastées sur le plan générationnel: d'un côté, des petits retraités (probablement d'anciens petits paysans ou membres des classes populaires ayant des ascendances ou alliances agricoles) poursuivant une micro-activité agricole (petits élevages, fruits ou maraîchage) sur un patrimoine hérité dans une perspective d'autoconsommation, de troc ou de complément de revenus (Laurent et al., 1998); de l'autre, des jeunes aux capitaux économique et culturel faibles découvrant la perspective d'un accès facilité au métier d'agriculteur par l'installation progressive (Malié et Nicolas, 2016).

\section{Quelle géographie des exploitations agricoles en transition?}

\subsection{Une géographie structurée par les orientations technico-économiques}

La répartition géographique des six types d'exploitation n'est pas homogène sur le territoire métropolitain. La figure 3 propose une première lecture géographique à l'échelon départemental. Trois ensembles géographiques se dessinent: l'Ouest français et l'Est du bassin parisien où dominent nettement et de longue date les exploitations conventionnelles en circuit long, un grand sud-est où l'agriculture biologique est bien ancrée, un sud-ouest où la cohabitation de circuits courts et longs sur une même exploitation (type B) est plus fréquente qu'ailleurs. Les orientations technico-économiques des exploitations (OTEX) structurent fortement cette géographie.

En 2010, les exploitations en agriculture biologique commercialisant en circuit long (type D) sont bien implantées sur la côte méditerranéenne et dans les départements de la basse vallée du Rhône, ainsi qu'en Aquitaine. Ces localisations privilégiées correspondent à la structuration en OTEX de ce type dominé à presque $30 \%$ par la viticulture, dont on reconnaît ici la géographie (Marie et al., 2015), surtout si l'on considère le pôle 


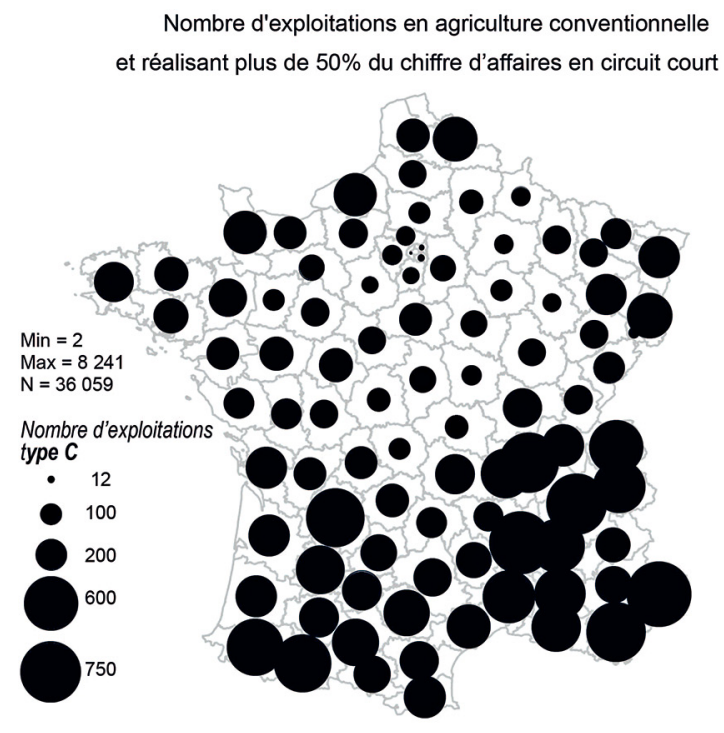

Nombre d'exploitations en agriculture biologique

et réalisant moins de $50 \%$ du hiffre d'affaires en circuit court (E)
Nombre d'exploitations en agriculture biologique

et commercialisant en circuit long (D)

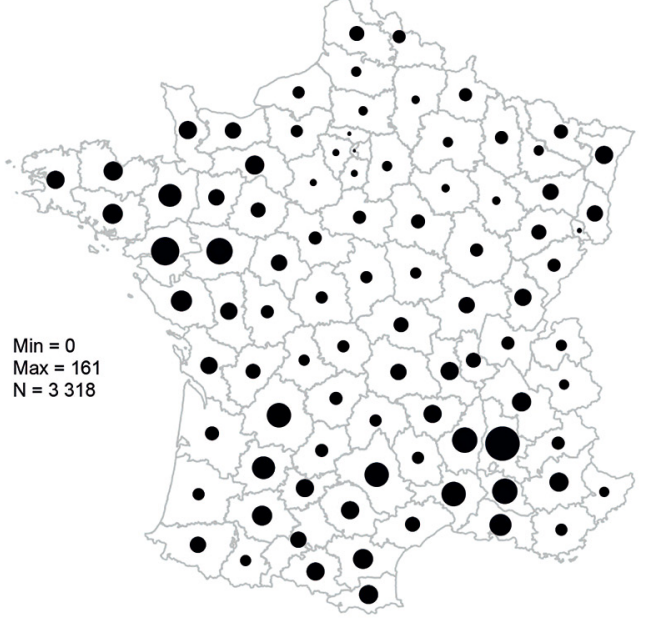

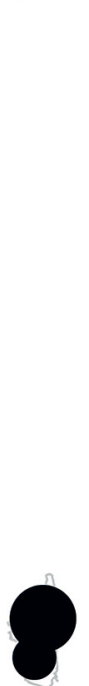

(E)

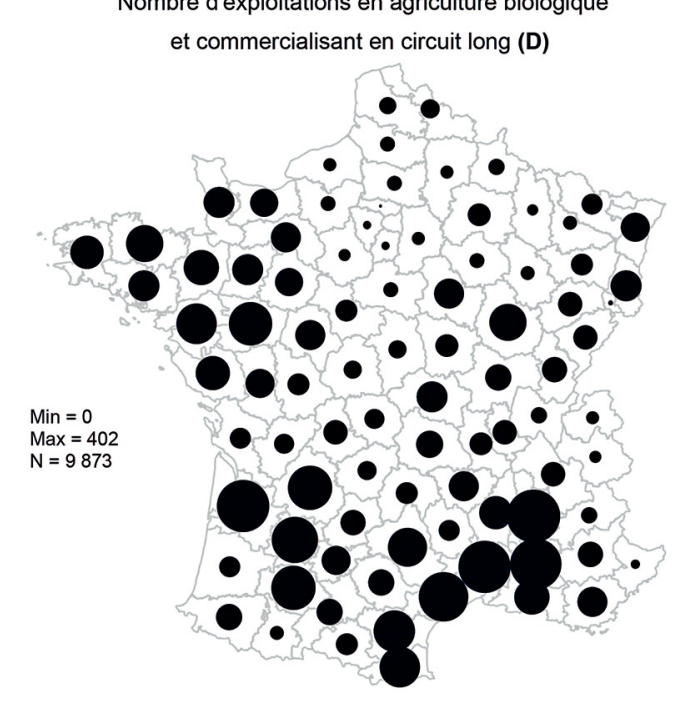

Nombre d'exploitations en agriculture biologique

et réalisant plus de $50 \%$ du chiffre d'affaires en circuit court (F)
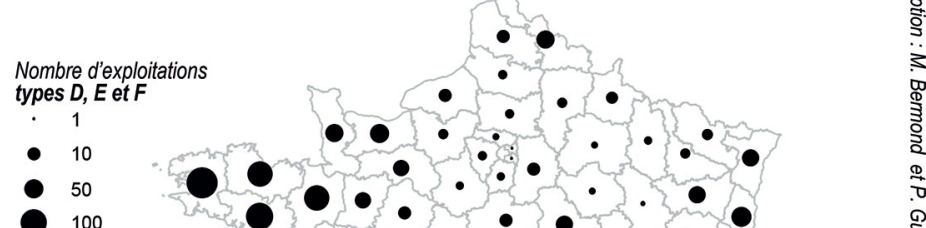

100

200
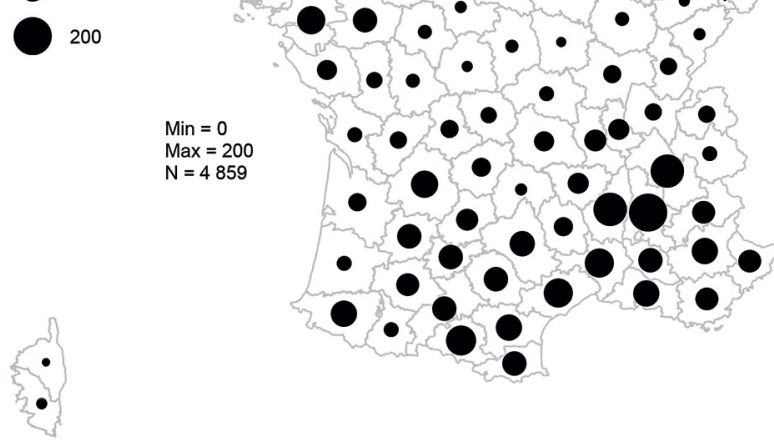

Min : valeur minimale; Max : valeur maximale ; $\mathrm{N}$ : effectif.

Fig. 3. Localisation des types d'exploitations agricoles en transition par département en 2010.

Fig. 3. Location of farm types in transition by Department in 2010.

alsacien (Fig. 3). Cette géographie se distingue en part de l'emploi agricole départemental, car la viticulture, indépendamment du label, nécessite une main-d'œuvre importante. Mais le type $\mathrm{D}$, bien doté en exploitations spécialisées bovins lait, est nettement représenté dans les campagnes de l'Ouest français où les combinaisons d'usages du sol agricole font la part belle aux systèmes principalement fourragers, avec aussi quelques vallées viticoles renommées (id.).

Avec de faibles effectifs et caractérisées par une diversité d'OTEX, les exploitations en agriculture biologique réalisant moins de $50 \%$ de leur chiffre d'affaires en circuit court (type E) se situent dans les Bouches du Rhône, le Gard, le Vaucluse, l'Ardèche, la Drôme, les Alpes-de-Haute-Provence et en Corse du Sud; en Loire-Atlantique, Maine-et-Loire et Deux-Sèvres; en Haute-Savoie et dans le Bas-Rhin. Pour le premier ensemble géographique, on retrouve les départements de prédilection des circuits courts et de l'agriculture biologique (Barry, 2012). D'une manière générale ressortent aussi des départements avec différentes combinaisons d'utilisation fourragère du sol agricole (Marie et al., 2015), du fait des OTEX structurant ce type: polyculture-polyélevage, bovins lait et bovins viande. Mais cette géographie recoupe aussi celle de la viticulture $(i d$.$) encore bien représentée pour le type E.$

Les fortes concentrations des exploitations agricoles conventionnelles réalisant plus de $50 \%$ du chiffre d'affaires en circuit court dans le sud de la France renvoient à la surreprésentation des circuits courts dans les anciennes régions Rhône-Alpes, Provence-Alpes-Côte-D'azur, LanguedocRoussillon, Midi-Pyrénées et Aquitaine (Barry, 2012). C'est encore à plusieurs combinaisons d'usages des sols agricoles 
qu'on est ici renvoyé : on retrouve à la fois des landes et estives et différentes formes de systèmes fourragers (id.) qui supportent des exploitations en polyculture-polyélevage alors que cette dernière OTEX et la spécialisation ovins-caprins représentent $40 \%$ des effectifs pour le type C. Les associations de vergers à la vigne et aux céréales sont surtout localisées dans la moitié sud de la France, terre d'élection des productions de légumes $(i d$.$) . Les fruits et autres cultures$ permanentes, ainsi que le maraîchage, totalisent presque un quart des exploitations du type C. En 2010, l'emploi agricole des départements d'Île-de-France ou Rhône-Alpes était aussi soutenu par ces exploitations conventionnelles et basées sur les circuits courts (Barry, 2012).

Avec des effectifs départementaux moindres, la même répartition géographique s'applique aux exploitations en agriculture biologique et réalisant plus de $50 \%$ du chiffre d'affaires en circuits courts (type F), qui ont une structuration en OTEX proche du type C (avec plus de maraîchagehorticulture, moins de polyculture-polyélevage). Le type F se distingue particulièrement dans les départements montagnards à forte attractivité touristique tels que l'Ardèche, l'Ariège, l'Isère, les Hautes-Alpes, les Alpes-de-Haute-Provence, les Alpes-Maritimes et la Haute-Corse.

\subsection{Combinaison géographique des formes de transition et contextes socio-territoriaux}

Bien qu'elle dégage de grandes tendances, cette approche géographique présente néanmoins deux limites: d'une part, elle traite de manière séparée les différents types de transition agricole, ne rendant pas aisée une analyse des combinaisons géographiques. Il serait intéressant de connaître les proximités et éloignements entre les types pour savoir s'il existe des combinaisons variables dans l'espace des types de transition agricole. Nous faisons l'hypothèse que les formes de transition agricole ne sont pas portées partout par les mêmes combinaisons de types. D'autre part, le niveau départemental d'analyse agglomère des situations locales parfois très contrastées: zones de plaine/zones de montagne, espaces ruraux de faibles densités en déprise démographique/espaces périurbains en forte croissance, économie fortement résidentielle ou touristiques/économie fortement agricole et industrielle. Les contextes démographiques et socio-économiques des territoires infléchissent également la nature des formes de transition agricole (Richard et al., 2014; Poulot et Rouyres, 2007).

C'est pourquoi nous proposons une analyse multidimensionnelle des types d'exploitations à l'échelon cantonal (Mignolet, 2005) pour rendre compte de l'hétérogénéité spatiale de l'agriculture en transition. La figure 4 présente les résultats d'une classification des cantons d'après le profil de répartition des exploitations selon la typologie des formes de transition agricole. Une analyse en composante principale (ACP - non présentée ici) sur le tableau cantonal de répartition des exploitations par types a fourni les coordonnées factorielles pour une classification ascendante hiérarchique des cantons sur les trois premiers facteurs $(86 \%$ de l'inertie totale). Une partition en sept groupes a été retenue. Seuls les cantons comptabilisant plus de dix exploitations au RA ont ici été retenus.
Un premier type géographique regroupe 838 cantons où les formes de transition agricole (telles que définies dans le cadre de cet article) sont quasi-inexistantes: les exploitations conventionnelles en filière longue représentent ici 9 exploitations sur 10 (contre 8 sur 10 en moyenne métropolitaine). Les exploitations biologiques sont quasi-inexistantes. On retrouve ici des zones rurales de faible densité où l'agriculture est fortement spécialisée et productive. Certains départements comme la Marne, l'Eure-et-Loir ou l'Indre sont quasiintégralement couverts par ce type.

Le deuxième type incarne le profil de répartition des exploitations le plus conforme à la moyenne métropolitaine : $80 \%$ d'exploitations conventionnelles en filière longue, $10 \%$ d'exploitations non biologiques recourant un peu aux circuits courts, $10 \%$ d'exploitations en transition. Le troisième type de cantons se rapproche du type 1 par une surreprésentation des exploitations conventionnelles en filière longue $(86,9 \%)$ et une sous-représentation des circuits courts parmi les conventionnels mais s'en distingue par l'émergence timide d'exploitations en transition ( $8 \%$ ). Le quatrième type s'inscrit toujours dans un contexte d'agriculture conventionnelle intégrée aux filières longues $(76 \%$ des exploitations) où la transition agricole prend la forme, soit d'une diversification vers des circuits courts sans conversion biologique, soit d'une conversion biologique intégrale hors développement des circuits courts.

Les trois derniers types de canton (types 5, 6 ou 7) sont ceux où les formes de transition agricole sont les plus avancées. Les exploitations conventionnelles en filière longue sont toujours inférieures à $60 \%$. Le type 5 se démarque par une surreprésentation significative de tous les types d'exploitation en transition. Une exploitation sur trois est classée en transition, aussi bien portée par le développement de circuits courts $(36 \%$ des exploitations) que par le mode de production biologique (19\% des exploitations), parfois les deux (10\% des exploitations). Les cantons de ce groupe sont essentiellement centrés sur le département de la Drôme, secondairement l'Ardèche et le sud des Cévennes - espaces emblématiques de la néo-ruralité. Le type 6 voit sa transition agricole quasi-exclusivement portée par des exploitations en circuit court sans que celles-ci optent pour le mode de production biologique. Deux éléments peuvent être avancés pour expliquer la faible conversion vers l'agriculture biologique: d'une part, ce type se localise pour partie dans des zones valorisant des appellations agricoles d'origine contrôlée $(\mathrm{AOC})$ ou protégées (AOP) où la plus-value agronomique et économique de l'agriculture biologique n'est pas décisive dans la stratégie de valorisation des produits agricoles (AOP-AOC laitière ou fromagère de moyenne montagne, Indications géographiques protégées de certains bassins de production spécialisés dans les légumes); d'autre part, ce type se localise en proche périphérie de certaines villes moyennes au contexte agricole standard (Châlons-sur-Saône, Laval, Metz, Caen, Limoges, Albi, Castres, etc.) qui peuvent être plutôt le siège d'exploitants professionnels développant de manière opportuniste des circuits courts pour des bassins urbains de consommation, ou bien encore le siège de populations néo-agricoles cherchant à satisfaire une demande urbaine en produits locaux (jeunes en phase de création d'une activité agricole alternative, petits retraités prolongeant une activité agricole de complément de revenu). 


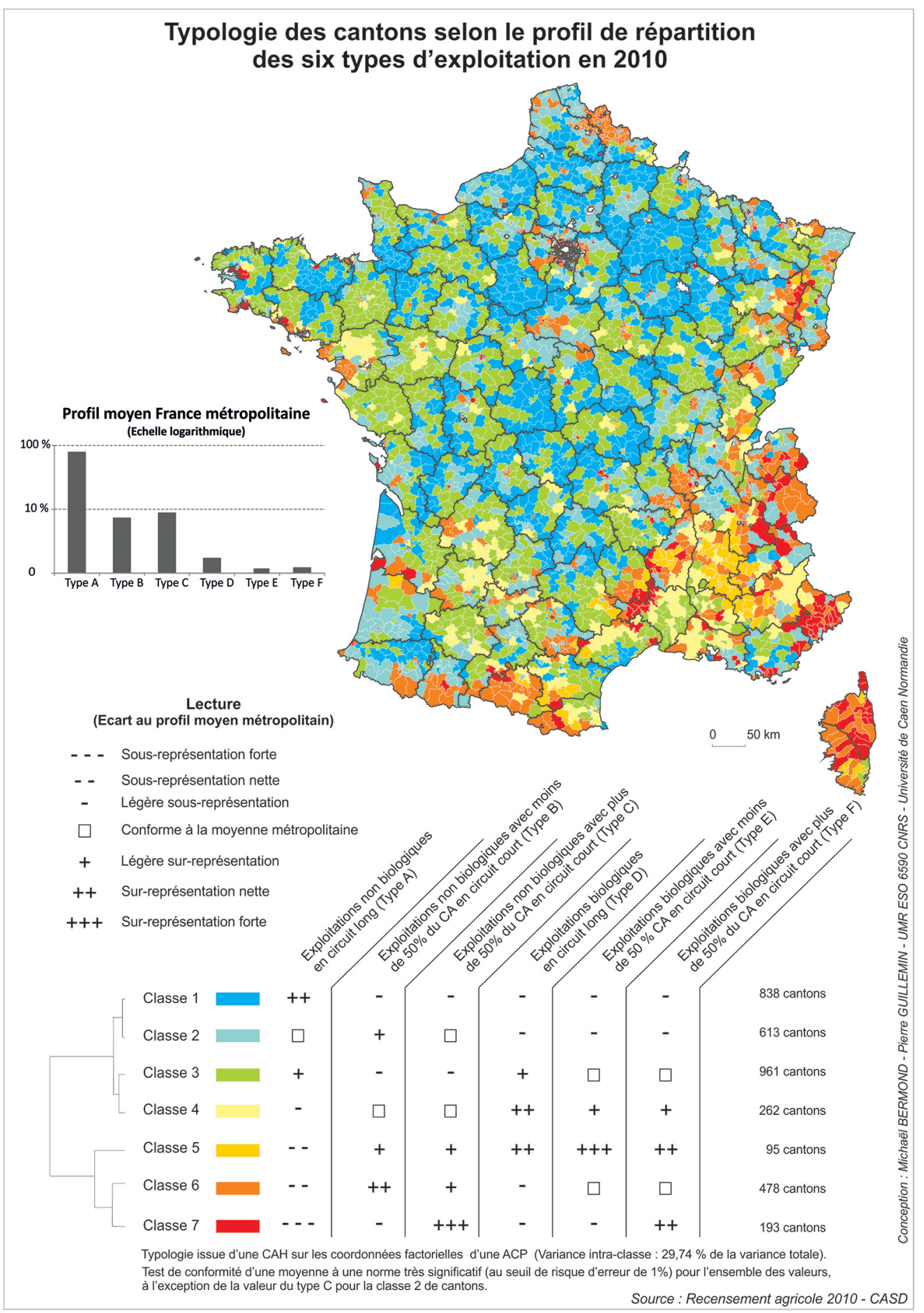

Fig. 4. Typologie des cantons selon les formes de transition agricole en 2010 en France.

Fig. 4. Typology of cantons according to the forms of agricultural transition in 2010 in France.

Le dernier type 7 présente de fortes parentés avec le type précédent, à ceci près que les exploitations en transition $(47 \%)$ sont ici quasiment à parité avec les exploitations conventionnelles en filière longue (48\%). Les exploitations non biologiques réalisant plus de $50 \%$ de leur chiffre d'affaire en circuit court représentent $40 \%$ du total. Leurs homologues en production biologique trouvent ici leur niveau le plus important avec $6 \%$ des exploitations (à peine $1 \%$ en moyenne métropolitaine). La proximité structurelle avec le type 6 se traduit aussi par une proximité géographique dans le prolongement de ce type. Ce type 7 isole également des zones urbaines (Angoulême, Poitiers, Biarritz, Bourg-en-Bresse, Bourges, Nevers, Troyes, etc.). 


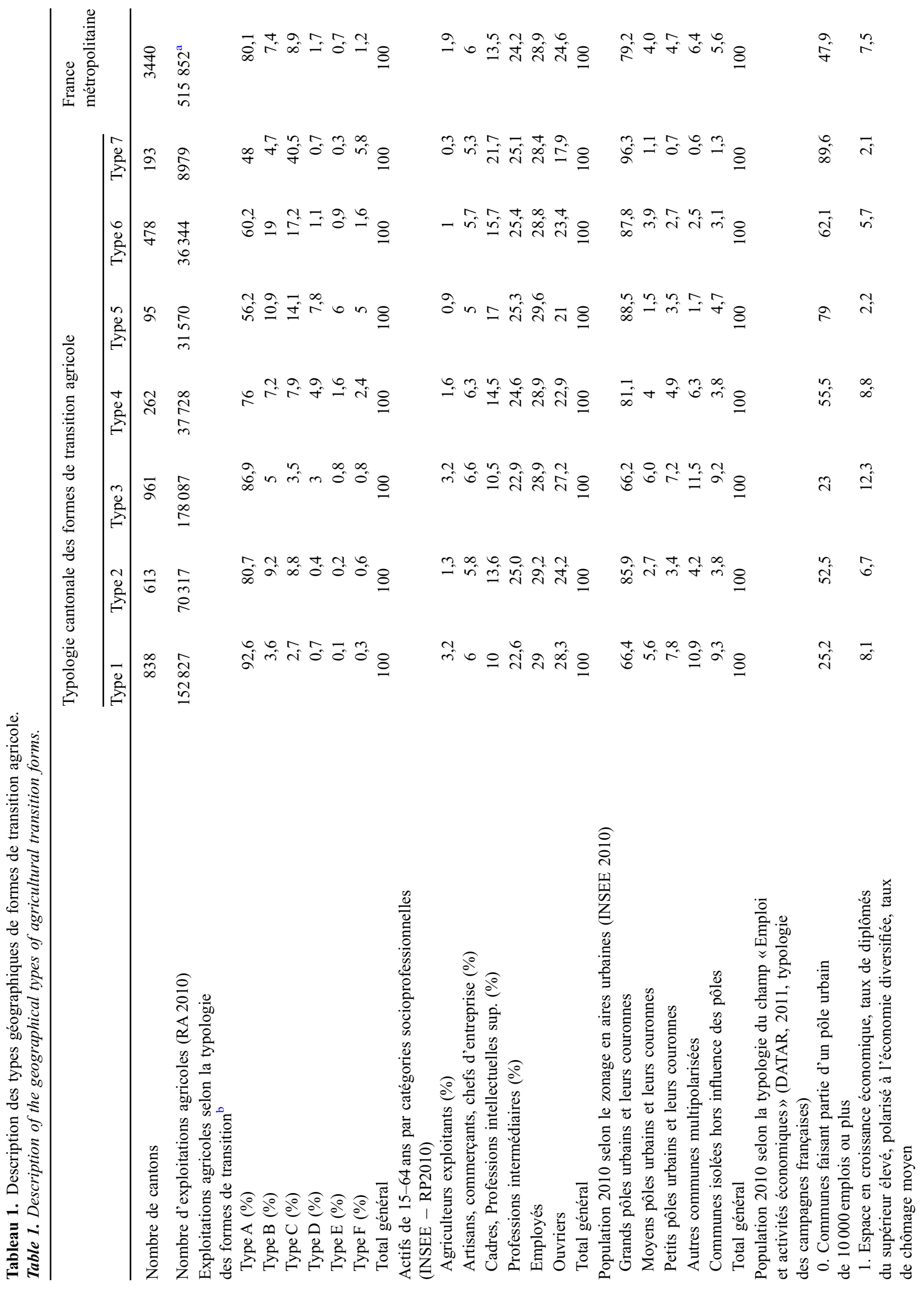


M. Bermond et al. : Cah. Agric. 2019, 28, 16

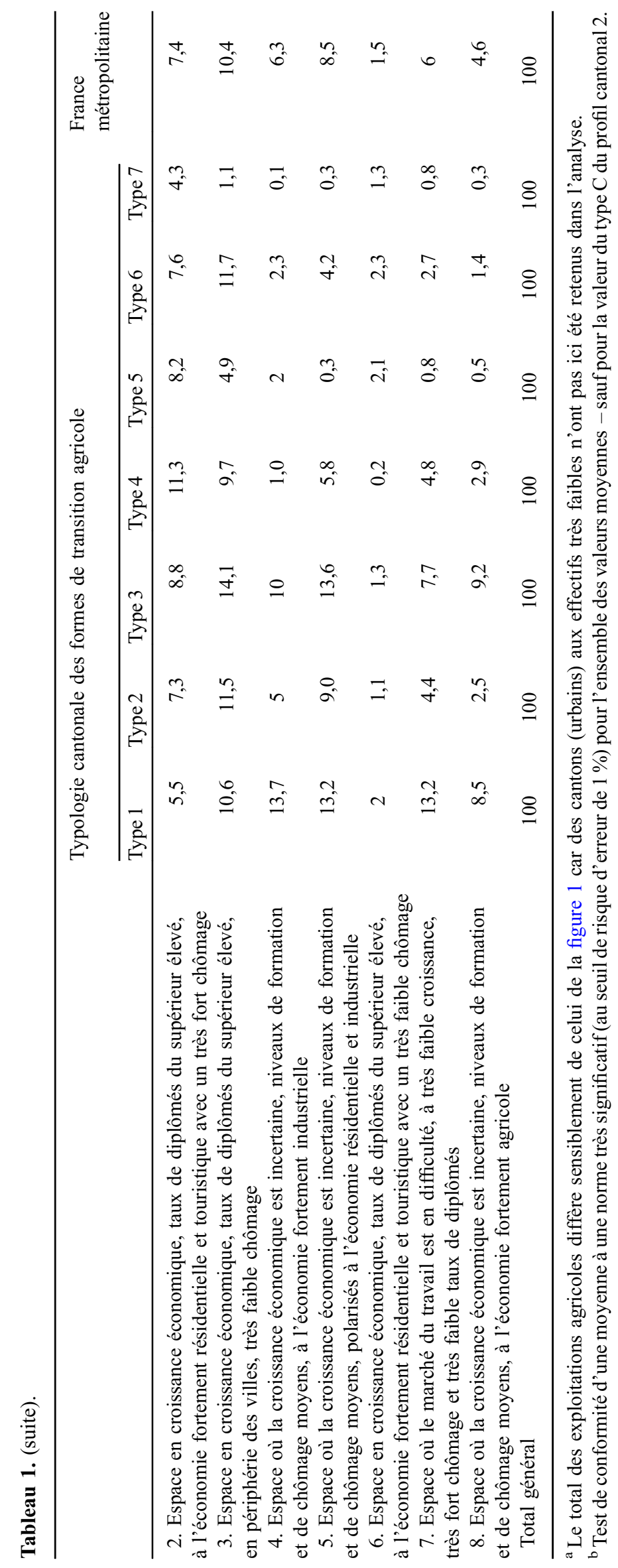

Page 10 de 13 
Au final se dessine une géographie opposant une France septentrionale et une France méridionale, mais aussi des périphéries urbaines et des espaces ruraux aux densités plus faibles. Si la géographie que dessine cette typologie emprunte des éléments explicatifs au contexte géo-agronomique des exploitations, elle suggère également que la nature du contexte socio-territorial dans lequel s'insèrent les exploitations infléchit les formes de transition agricole. Les espaces ruraux les moins dynamiques et les plus marqués par l'héritage du modèle productiviste restent dans leur grande majorité les plus résistants à l'émergence des formes de transition. Le tableau 1 montre bien que c'est au sein du type 1 que, non seulement la part des populations résidant dans les aires urbaines (au sens du zonage de l'INSEE de 2015) est la plus faible, mais que la part de la population vivant dans les espaces les plus fragiles démographiquement et économiquement (au sens de la typologie des campagnes de la DATAR; Hilal et al., 2011) est la plus forte. À l'inverse, les types d'exploitations les plus engagées dans les formes de transition ne se rencontrent pas seulement dans les régions tournées de longue date vers des produits agricoles à plus forte valeur ajoutée (vins, produits laitiers sous AOP-AOC, productions sous labels); ils se rencontrent aussi dans des espaces où le profil de la population résidente est socialement et économiquement le plus réceptif aux circuits courts, suggérant un effet de proximité géographique entre certaines agricultures en transition et populations résidentes aux profils plus aisés (Chevalier et al., 2014). La part de la population active résidente appartenant à la catégorie socioprofessionnelle des cadres et professions intellectuelles est la plus élevée dans les cantons des types $5(17 \%)$ et $7(21,7 \%)-$ cantons largement urbanisés ou dont les communes rurales sont majoritairement classées d'après la typologie des campagnes de la DATAR (2012) parmi les espaces en forte croissance économique avec un taux de diplômés du supérieur élevé (tableau 1). La proximité géographique avec des populations plus aisées ne saurait cependant épuiser la réalité des liens entre agriculture en transition et espaces sociaux plus aisés, comme en témoigne la corrélation, certes positive mais néanmoins d'intensité moyenne $(+0,44)$, entre la part des populations appartenant aux catégories socioprofessionnelles supérieures et la part des exploitations en transition à l'échelon des cantons métropolitains. Ces résultats invitent à creuser la multiplicité des facteurs qui interviennent dans l'émergence d'un marché alimentaire d'approvisionnement local.

Les résultats obtenus invitent enfin à ne pas perdre de vue que le choix de critères complémentaires pour couvrir plus largement le champ des agricultures en transition aurait sensiblement modifié les représentations cartographiques. Par exemple, la prise en compte de critères permettant d'identifier l'agriculture de conservation - phénomène non négligeable en France (si on considère tout le gradient allant des Techniques Culturales Simplifiées à la conservation des sols) - aurait redonné davantage de poids aux systèmes agricoles du nord de la France dans l'analyse spatiale des formes de transition agroécologique (Laurent, 2015). Cette observation renforce l'idée que les formes d'agriculture en transition sont le produit historique d'un agencement variable dans l'espace de facteurs structurels et contextuels favorables à leur développement.

\section{Conclusion}

La présente contribution propose une approche quantitative et cartographique exploratoire de certaines formes d'agriculture en transition sur le territoire métropolitain français. À partir du Recensement agricole 2010, six types d'exploitations ont été identifiés selon un gradient d'écologisation des pratiques prenant en compte le mode de production (biologique ou non) et le mode de commercialisation des produits. Quatre types ont été retenus comme pouvant délimiter potentiellement un champ statistique de l'agriculture en transition. S'ils ont en commun une intensité du travail plus élevée, ils présentent des caractéristiques socio-économiques bien différenciées.

Ces types d'agriculture en transition dessinent à l'échelon local une géographie complexe où se combinent des facteurs sectoriels (adaptation des systèmes aux conditions pédoclimatiques, politiques agricoles), socio-historiques (poids des filières et des circuits de distribution alimentaire, histoire des systèmes de production, importance des réseaux agricoles alternatifs, etc.) et des facteurs liés à l'environnement sociogéographique (proximités urbaines, zones touristiques, niveau socio-économique des populations résidentes, etc.). Les résultats obtenus reposent sur un choix de définition des exploitations en transition qui mériterait d'être élargi afin d'intégrer d'autres formes de transition agro-écologique non étudiées ici (agriculture de conservation, agro-foresterie, permaculture...).

En cartographiant de manière fine certains types d'agriculture en transition, notre travail invite à poursuivre les réflexions sur les liens complexes unissant agriculture en transition et espace. Bon nombre de travaux scientifiques relatifs aux facteurs de développement des formes d'agriculture en transition intègrent dans leurs analyses la prise en compte des innovations territoriales. Pour certains, les discours sur la relocalisation de la production agricole sont en partie le résultat d'une territorialisation des politiques publiques agricoles (Amemiya, 2011). Pour d'autres, ces innovations territoriales s'inscrivent dans une problématique plus large de développement local (Marsden et al., 1999; Chiffoleau et Prevost, 2012). La présente contribution rappelle que certaines formes de transition agro-écologique peuvent être aussi portées par des contextes géographiques où le profil socioéconomique des populations résidentes rend possible des alliances culturelles avec des producteurs «innovants» dont les circuits courts peuvent être le support.

Remerciements. Cette étude s'inscrit dans le cadre des recherches développées par le projet FRUGAL (Forme Urbaine et Gouvernance Alimentaire). Elle a bénéficié à ce titre du soutien du programme PSDR 4 (Pour et Sur le Développement Régional) financé par l'INRA (Institut National de Recherche Agronomique), l'IRSTEA (Institut national de Recherche en Sciences et Technologie pour l'Environnement et l'Agriculture) et les régions Bretagne, Pays de la Loire, Normandie, Nouvelle-Aquitaine et Auvergne-Rhône-Alpes.

Ce travail a également bénéficié d'une aide de l'État gérée par l'Agence Nationale de la Recherche au titre du programme Investissements d'avenir portant la référence ANR-10-EQPX17 (Centre d'accès sécurisé aux données - CASD). 
Nous remercions Jean-Pascal Guironnet, enseignantchercheur au centre de recherche en économie et management (CREM - UMR 6211 CNRS) de l'Université de Caen Normandie pour ses recommandations sur la mise en œuvre des tests de significativité statistique.

Nos remerciements les plus sincères vont enfin aux deux relecteurs anonymes qui ont permis, par leurs conseils avisés, d'enrichir et finaliser cet article.

\section{Références}

Amemiya H. 2011. Du Teikei aux AMAP. Le renouveau de la vente directe de produits fermiers locaux. Rennes: Presses Universitaires de Rennes, 350 p.

Aubry C, Bressoud F, Petit C. 2011. Les circuits-courts en agriculture revisitent-ils l'organisation du travail dans l'exploitation? In: Beguin P, Dedieu V, Sabourin E (Dir.). Le travail en agriculture: son organisation et ses valeurs face à l'innovation. Paris: L'Harmattan.

Bâ M, Gresset-Bourgeois M, Quirion P. 2016. L'effet sur l'emploi d'une transition écologique sur l'agriculture en France. Le cas du scénario Afterres. Le Courrier de l'environnement de l'INRA 66: 93-102.

Barry C. 2012. Un producteur sur cinq vend en circuit court. Agreste Primeurs 275: 4 p.

Chantre E, Cardona A. 2014. Trajectories of French Field Crop Farmers moving toward sustainable farming practices: change, learning, and links with the advisory services. Agroecology and Sustainable Food Systems 38(5): 573-602. DOI: 10.3390/ su10062083.

Chevalier M, Dellier J, Plumecocq G, Richard F. 2014. Dynamiques et structuration des circuits courts agroalimentaires en Limousin: distance institutionnelle, proximités spatiale et relationnelle. Géographie, économie et société 16: 339-362. DOI: 10.3166/ ges.16.339-362.

Chiffoleau Y, Prevost B. 2012. Les circuits courts, des innovations sociales pour une alimentation durable dans les territoires. Norois 224: 7-20. DOI: 10.4000/norois.4245.

Dedieu MS, Lorge A, Louveau O, Marcus V. 2017. Les exploitations en agriculture biologique: quelles performances économiques? Les acteurs économiques et l'environnement. Insee Références, 3544.

Desriers M. 2007. L'agriculture française depuis cinquante ans : des petites exploitations aux droits à paiement unique. Agreste Cahiers 2: $14 \mathrm{p}$.

Deverre C, Lamine C. 2010. Les systèmes agroalimentaires alternatifs. Une revue de travaux anglophones en sciences sociales. Economie rurale 317: 57-73. DOI: 10.4000/economierurale.2676.

Dufour A, Giraud C. 2012. Le travail dans les exploitations d'élevage bovin laitier est-il toujours conjugal? INRA Productions animales 25(2): 169-179.

Dugué P, Djamen Nana P, Faure G, Le Gal PY. 2015. Dynamiques d'adoption de l'agriculture de conservation dans les exploitations familiales: de la technique aux processus d'innovation. Cahiers Agricultures 24(2): 60-68. DOI: 10.1684/agr.2015.0748.

Duru M, Fares M, Therond O. 2014. Un cadre conceptuel pour penser maintenant (et organiser demain) la transition agroécologique de l'agriculture dans les territoires. Cahiers Agricultures 23: 84-95. DOI: 10.1684/agr.2014.0691.

Elzen B, Augustyn AM, Barbier M, Van Mierlo B. 2017. AgroEcological Transitions. Wageningen: Wageningen University.
Fares M, Magrini M, Triboulet P. 2012, Transition agroécologique, innovation et effets de verrouillage : le rôle de la structure organisationnelle des filières. Le cas de la filière blé dur française. Cahiers Agricultures 21: 34-45. DOI: 10.1684/agr.2012.0539.

Féret S, Douguet JM. 2001. Agriculture durable et agriculture raisonnée. Quels principes et quelles pratiques pour la soutenabilité du développement en agriculture? Natures, Sciences, Sociétés 9(1): 58-64. DOI: 10.1016/S1240-1307(01)90012-3.

Garambois N. 2011. Des prairies et des hommes. Les systèmes herbagers économes du bocage poitevin. Agro-écologie, création de richesse et emploi en élevage bovin. Thèse de doctorat, AgroParisTech : 595 p.

Griffon M. 2013. Qu'est-ce que l'agriculture écologiquement intensive? Paris: Édition Quae 2013, Collection: Matière à débattre et décider, $224 \mathrm{p}$.

Hilal M, Barczak A, Tourneu FP, Schaeffer Y, Houdart M, CremerSchulte D. 2011. Typologie des campagnes françaises et des espaces à enjeux spécifiques (littoral, montagnes et DOM). DATAR, 78 p. https://hal.archives-ouvertes.fr/hal-00911232.

Kroll JC. 1990. Agriculture: changer de politique. Paris: Syros, collection Alternatives Économiques, 194 p.

Lamine C, Bellon S. 2009. Transitions vers l'agriculture biologique. Pratiques et accompagnements pour des systèmes innovants. Paris: éd. Éducagri-Quae, 316 p.

Lamine C, Renting H, Rossi A, Han Wiskerke JSC, Brunori G. 2012. Agri-Food systems and territorial development: innovations, new dynamics and changing governance mechanism. Farming Systems Research into the 21 st Century: The New Dynamics. Netherlands: Springer, pp. 229-256. DOI: 10.1007/978-94-007-4503-2_11.

Laurent C, Cartier S, Fabre C, Mundler P, Ponchelet D, Rémy J. 1998. L'activité agricole et la cohésion économique et sociale. Economie rurale 244: 134-152. DOI: 10.3406/ecoru.1998.4996.

Laurent C, Maxime F, Mazé A, Tichit M. 2003. Multifonctionnalité de l'agriculture et modèles de l'exploitation agricole. Économie rurale 273-274: 134-152. DOI: 10.3406/ecoru.2003.5395.

Legagneux B, Olivier-Salvagnac V. 2017. Quelle main-d'œuvre contractuelle dans les exploitations agricoles? À la base de l'éclatement du modèle familial. Economie Rurale 357-358: 101122. DOI: $10.4000 /$ economierurale.5132.

Levaux MB, Genty B. 2015. L'emploi dans la transition écologique. Avis du Conseil Économique, Social et Environnemental, 127 p.

Mahé T, Lerbourg J. 2012. Des agriculteurs bio diplômés, jeunes et tournés vers les circuits courts. Agreste Primeurs 284: 4 p.

Malié A, Nicolas F. 2016. Des loisirs aux «alternatives ». Le rapport ambivalent des classes populaires aux pratiques agricoles et alimentaires en milieu rural. Revue Savoir/agir 38: 37-43. DOI: $10.3917 /$ sava.038.0037.

Mansfield B, Mendes W. 2013. Municipal food strategies and integrated approaches to urban agriculture: exploring three cases from the Global North. International Planning Studies 18-1: $37-$ 60. DOI: $10.1080 / 13563475.2013 .750942$.

Marie M, Bermond M, Madeline P, Coinaud C. 2015. Une typologie des combinaisons d'utilisation agricole du sol en France en 2010 : propositions méthodologiques. Mappemonde 114: 24 p.

Maréchal G, Spanu A. 2010. Les circuits courts favorisent-ils l'adoption de pratiques agricoles plus respectueuses de l'environnement ? Le Courrier de l'environnement de l'INRA 59: 33-45.

Marsden T, Murdoch J, Morgan K. 1999. Sustainable agriculture, food supply chains and regional development. International Planning Studies 3: 295-301. DOI: 10.1080/13563479908721743.

Massis D, Hild F. 2016. La pratique de l'agriculture biologique créatrice d'emploi? Une évaluation de l'impact du bio sur la quantité de travail agricole. Agreste Les Dossiers 35: 32 p. 
Mignolet C. 2005. Diversité régionale des exploitations agricoles et de leurs dynamiques: méthode de spatialisation. In : Laurent $\mathrm{C}$, Thinon P (Coord.), Agricultures et territoires. Édition Lavoisier, pp. $115-133$.

Moragues-Faus A. 2016. Revisiting food studies from a political ecology perspective: lessons from Mediterranean agri-food systems. Agriculture, Environnement and Development. Cham. Springer: pp. 59-90.

Plumecocq G, Debril T, Duru M, Magrini MB, Sarthou JP, Theron O. 2018. Caractérisation socio-économique des formes d'agriculture durable. Économie rurale 363: 99-120. DOI: 10.4000/economie rurale. 5430 .

Poulot M, Rouyres T. 2007. Refaire campagne en Île-de-France. Norois 202: 61-71. DOI: 10.4000/norois.1631.

Rémy J. 1990. Qui est agriculteur? In: Coulomb P, Delorme H, Hervieu B, Jollivet M, Lacombe P (dir.). Les agriculteurs et la politique. Paris: Presses de Sciences Po, pp. 257-265.

Roy C, Teyssier d'Orfeuil J. 2016. Dynamiques de l'emploi dans les filières bioéconomiques. Rapport $n^{\circ} 15056$ du Conseil Général de l'Alimentation et de l'Agriculture et des Espaces Ruraux. 63 p.

Laurent F. 2015. L'agriculture de conservation et sa diffusion en France et dans le monde. Cybergeo. European Journal of Geography [En ligne], Environnement, Nature, Paysage, document 747. DOI: $10.4000 /$ cybergeo. 27284 .

Renting H, Wiskerke JSC. 2010. New emerging role for public institutions and civil society in the promotion of sustainable local agro-food systems. 9th European IFSA Symposium: Vienne, Autriche, pp. 1902-1912.

Richard F, Chevallier M, Dellier J, Lagarde V. 2014. Circuits courts agroalimentaires de proximité en Limousin: performance économique et processus de gentrification rurale. Norois 230: 21-39. DOI: $10.4000 /$ norois.4997.

Samak M. 2016. La politisation variable des alternatives agricoles. Revue Savoir/agir 38: 29-35. DOI: 10.3917/sava.038.0029.

Sonnino R. 2014. The new geography of food security: exploring the potential of urban food strategies. The Geographical Journal 182-2: 190-200. DOI: 10.1111/geoj.12129.

Tavernier Y, Gervais M, Servolin C. 1972. L'univers politique des paysans dans la France contemporaine. Paris: Armand Colin, $653 \mathrm{p}$.

Therond O, Duru M, Roger-Estrade J, Richard G. 2017. A new analytical framework of farming system and agriculture model diversities. A review. Agronomy for Sustainable Development 37: 21. DOI: $10.1007 / \mathrm{s} 13593-017-0429-7$.

Wezel A, Brives H, Casagrande M, Clément C, Dufour A, Vandenbroucke P. 2016. Agroecology territories: places for sustainable agricultural and foodsystems and biodiversity conservation. Agroecology and Sustainable Food Systems 40: 132-144. DOI: $10.1080 / 21683565.2015 .1115799$.

Wiskerke JSC. 2009. On place lost and places regained: reflections on the alternative food geography and sustainable regional development. International Planning Studies 14-4: 369-387. DOI: 10.1080/13563471003642803.

Citation de l'article : Bermond M, Guillemin P, Maréchal G. 2019. Quelle géographie des transitions agricoles en France ? Une approche exploratoire à partir de l'agriculture biologique et des circuits courts dans le recensement agricole 2010. Cah. Agric. 28 : 16. 